\title{
Validation of administrative data sources for endoscopy utilization in colorectal cancer diagnosis
}

\author{
Xue Li ${ }^{1}$, Robert Hilsden ${ }^{2}$, Shakhawat Hossain ${ }^{3,4}$, John Fleming ${ }^{1}$ and Marcy Winget ${ }^{1,3^{*}}$
}

\begin{abstract}
Background: Validation of administrative data is important to assess potential sources of bias in outcome evaluation and to prevent dissemination of misleading or inaccurate information. The purpose of the study was to determine the completeness and accuracy of endoscopy data in several administrative data sources in the year prior to colorectal cancer diagnosis as part of a larger project focused on evaluating the quality of pre-diagnostic care.

Methods: Primary and secondary data sources for endoscopy were collected from the Alberta Cancer Registry, cancer medical charts and three different administrative data sources. 1672 randomly sampled patients diagnosed with invasive colorectal cancer in years 2000-2005 in Alberta, Canada were included. A retrospective validation study of administrative data for endoscopy in the year prior to colorectal cancer diagnosis was conducted. A gold standard dataset was created by combining all the datasets. Number and percent identified, agreement and percent unique to a given data source were calculated and compared across each dataset and to the gold standard with respect to identifying all patients who underwent endoscopy and all endoscopies received by those patients.
\end{abstract}

Results: The combined administrative data and physician billing data identified as high or higher percentage of patients who had one or more endoscopy ( $84 \%$ and $78 \%$, respectively) and total endoscopy procedures (89\% and $81 \%$, respectively) than the chart review (78\% for both).

Conclusions: Endoscopy data has a high level of completeness and accuracy in physician billing data alone. Combined with hospital in/outpatient data it is more complete than chart review alone.

Keywords: Colorectal endoscopy, Data validation, Administrative data, Agreement

\section{Background}

Databases that are developed and maintained for administrative purposes are frequently used in population health research and disease surveillance because of their availability, generality, cost-effectiveness and large population encompassed. The quality of administrative data, however, is often questioned when the data are employed in health outcomes research or quality measurement [1-9]. It is,

\footnotetext{
* Correspondence: marcy.winget@albertahealthservices.ca

'Division of Community Oncology, Cancer Care, Alberta Health Services, Edmonton, Alberta, Canada

${ }^{3}$ Department of Public Health Sciences, School of Public Health, University of Alberta, 1500 Sun Life Place, 1012399 Street NW, Edmonton, Alberta T5J $3 \mathrm{H} 1$, Canada

Full list of author information is available at the end of the article
}

therefore, important to validate administrative data in order to assess potential sources of bias in outcome evaluation and to prevent dissemination of misleading or inaccurate information [10].

Validation studies of administrative data have primarily focused on diagnosis of disease [10-20]. In cancer research, however, the primary data source used for identifying cancer cases is typically a well-established cancer registry; administrative data are not usually used or needed to identify cancer cases. Administrative data, however, can be very valuable in identifying key procedures received during a cancer patient's care trajectory in order to evaluate the care received [21,22], to understand patterns of service delivery [23], and/or to predict

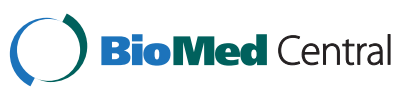


future resource needs [24]. Validating the potential administrative data sources to be used in such studies should be a critical component of the study itself.

The purpose of the study was to validate the completeness and accuracy of endoscopy data in several administrative data sources in the year prior to colorectal cancer diagnosis as part of a larger project focused on evaluating the quality of the pre-diagnostic care trajectory of colorectal cancer patients with respect to tests received and timing of them.

\section{Methods}

\section{Inclusion criteria}

An approximate 20\% random sample of all residents of Alberta, Canada diagnosed with invasive colon cancer (International Classification of Diseases for Oncology (ICD-O) [25] codes: c18, excluding appendix) or rectal cancer (ICD-O c19 and c20) in years 2000 to 2005, stratified by stage and year of diagnosis, were identified from the Alberta Cancer Registry and included in the study. Patients were excluded for the following reasons: stage 0 cancer; histology that are not staged according to the Collaborative Staging Guidelines [26]; or missing the unique lifetime identifier (ULI). The ULI is a unique number assigned to all members of the Alberta Health Care Insurance Program (AHCIP), the publicly-funded provincial healthcare insurance plan in Alberta. The ULI is, therefore, used as the anonymized patient identifier in all provincial administrative databases in Alberta and was used to link data across data sources for the study.

\section{Chart review data}

A chart review using the cancer clinic medical chart was conducted to identify dates of endoscopy prior to and including the date of diagnosis. Cancer medical charts are initially created for all patients diagnosed with cancer by the Alberta Cancer Registry for use in coding cases. They include procedure reports such as those for pathology, surgery, or endoscopy, plus referral letters and dictation notes, if the patient is seen by an oncologist; thus a cancer chart exists for every patient diagnosed with cancer in the province. The following data were abstracted from the charts: date and type of endoscopy; result (cancer, suspicious, not cancer); and source of information (letter, dictation notes, report).

\section{Administrative health databases}

Endoscopy data were obtained from three provincial administrative databases, the first two of which conform to national reporting standards: 1) the Discharge Abstract Database (hospital inpatient data) which records information on all admissions to hospitals in Alberta; 2) the Ambulatory Care Classification System Database (hospital outpatient data), which contains information on all outpatient visits that occurred in hospitals, such as visits to hospital-based physicians' offices, hospital endoscopy units, and emergency departments; and 3) the Physician Billing database, which contains all billing claims submitted by physicians remunerated on a fee-for-service basis and "shadow" billing submitted by physicians employed through the Alternate Relationship Plan (ARP). The latter group of physicians comprises a small number of physicians in one city during the time period of this study. From each data source, dates and codes for endoscopy procedures were identified that occurred within one year prior to colorectal cancer diagnosis for each patient included in the study. The timeframe of one year prior to diagnosis was determined based on a sensitivity analysis we conducted comparing endoscopies found 12, 18, or 24 months prior to colorectal cancer diagnosis; roughly the same number were found regardless of the time frame, therefore we used one year as the cutoff.

Each data source uses a different coding system and coding systems changed from ICD-9 to ICD-10 in April 2002 for the hospital datasets. In order to identify endoscopy codes from each data source appropriately, a literature review was conducted and input from local physicians was obtained. Since our purpose was to identify all lower gastrointestinal endoscopies regardless of purpose, all codes that indicated use of an endoscope were included. The endoscopy procedure codes included in the study from each data source are listed in Additional file 1.

\section{Combined administrative dataset}

The three administrative datasets were combined using the assumption that if an endoscopy was identified in any source then it was assumed to have occurred. This is because: 1) we expect that most patients will have had an endoscopy prior to colorectal cancer diagnosis and 2) it is unlikely that an endoscopy would be identified in any of the data sources if it was not actually performed; that is, the probability of a false positive is low. The data were combined in such a way as to minimize error in identifying unique endoscopies and also to assess accuracy with respect to the date of the endoscopy in the various data sources. In practice, it would be reasonable for an endoscopy code for the same event to appear in a hospital inpatient record and physician billing record or hospital outpatient record and physician billing record. Coding rules and practices should prevent the same event from being coded in both hospital inpatient and outpatient data unless an error is made. This is because procedures that happen to patients as outpatients should not be entered as a procedure as an inpatient (and vice versa), even if the patient is admitted the same day. Similarly, it is unlikely that a patient would undergo more than one endoscopy on the same day. Furthermore, dates for events in the hospital databases are 
expected to be accurate because the data are entered and coded by trained health records technicians. Physician billing, however, is more prone to error with respect to both the accuracy of the code and the date. In order to minimize the chance of counting a given endoscopy more than once and minimize the chance of counting two or more events as one when combining the datasets, the following rules were applied: 1) if an endoscopy appeared in both the inpatient and outpatient datasets for the same individual and date it was considered to be the same endoscopy; 2) if an endoscopy in the physician billing data was within three days of an endoscopy in either hospital dataset then it was counted as the same endoscopy. These rules were tested against rules using three and seven day windows, respectively, with the result that there was minimal difference in the number of unique endoscopies identified. If a patient did not appear in a dataset then the patient was assigned to the "No Endoscopy" category for that particular dataset.

\section{Gold standard}

The gold standard dataset was created by combining all administrative datasets and the chart review data. If a procedure was identified in any data set, it was considered to have occurred in the gold standard. The cancer clinic medical chart was not adopted as the gold standard because, even though information that is collected by the cancer registry to code and stage patients is in these charts, it is possible that an endoscopy that did not result in removal of tissue would be missed. Furthermore, although pathology reports are obtained when possible, some information may be obtained from referral letters or dictation notes which are subject to error. For this reason a gold standard was created to maximize the probability of identifying all unique endoscopies conducted in the year prior to colorectal cancer diagnosis. The same rules and assumptions that were followed to create the combined administrative dataset were applied in creating the gold standard: 1) if an endoscopy appeared in either data source then it was assumed to have occurred (probability of false-positive is low) and 2) endoscopies in the chart review dataset that were within three days of the date of an endoscopy in the combined administrative dataset were counted as the same endoscopy.

\section{Data analysis}

The measures to evaluate the completeness of the data were calculated at two levels: 1) comparing the total number of patients that underwent endoscopy and 2) comparing the total number of endoscopy procedures identified in each data source. The following descriptive statistics were calculated regarding patients who received an endoscopy and endoscopies identified from each dataset using the respective totals identified in the gold standard as the denominators for percentages: 1) total number and percent, 2) the number and percent identified from one and only one data source, by data source and, 3) the number and percent identified from one and only one of the administrative data sources, by administrative data source; note, these may have also been identified from the chart review. The purpose of this latter set of statistics is to indicate the extent to which each administrative data source contributes uniquely in the absence of a chart review. The percentage of endoscopy procedures that had exact date matches was used to determine the accuracy of the data.

In order to assess the likelihood that endoscopies were missed, clinical characteristics and health care service utilization were compared between patients who had an endoscopy to those who did not. Specifically, patient age at diagnosis, disease stage, type of first colorectal cancerrelated healthcare visit (pre-diagnostic or not), and time from diagnosis to death were explored. These were selected because they were considered to be potentially relevant reasons individuals may not receive an endoscopy prior to colorectal cancer diagnosis. Statistical significance was defined at the $\alpha=0.05$ level. All analyses were performed using statistical software SAS 9.1.3 (SAS Institute, Cary, NC, USA) or STATA/SE 10.0 (StataCorp LP, TX, USA).

\section{Results}

There were 1672 patients diagnosed with colorectal cancer in years 2000-2005 who were randomly selected and included in the study. Table 1 compares the patient characteristics and health service utilization in the entire population of colorectal cancer patients diagnosed in Alberta in years 2000-2005 versus the sample of 1672 patients. The sample of patients included in the study is representative of the population on the factors examined.

Table 2 describes the endoscopy data obtained from the chart review. There were 1506 endoscopies identified from the patient charts. Over half (65\%) of the data were abstracted from pathology reports, nearly $30 \%$ of the endoscopies were sigmoidoscopies, and the results for $93 \%$ of the endoscopies were a cancer diagnosis.

Table 3 summarizes the total number of patients and endoscopy procedures identified from each data source relative to the gold standard and the number and percent that were uniquely identified from each data source. Out of 1672 patients included in the study, a total of 1937 endoscopy procedures conducted on 1443 patients (86\%) were identified by the gold standard. The combined administrative data identified 1732 (89\%) of the endoscopy procedures and 1403 (84\%) of the patients, this was somewhat higher than the endoscopies (1506, $78 \%$ ) and patients who had an endoscopy (1310, 78\%) identified by chart review alone. The physician billing 
Table 1 Patient characteristics of cohort and sample

\begin{tabular}{|c|c|c|}
\hline Characteristics & Cohort (\%) & Sample (\%) \\
\hline Total Number & $8310(100)$ & $1672(100)$ \\
\hline \multicolumn{3}{|l|}{ Age at diagnosis } \\
\hline Mean [Median] & $69[70]$ & $69[70]$ \\
\hline$<=65$ & $3042(37)$ & $643(38)$ \\
\hline $66-74$ & $2172(26)$ & $437(26)$ \\
\hline$>=75$ & $3096(37)$ & $592(35)$ \\
\hline \multicolumn{3}{|l|}{ Stage at diagnosis } \\
\hline$|-|||$ & $5500(66)$ & $1145(68)$ \\
\hline IV or Unknown & $2810(34)$ & $527(32)$ \\
\hline \multicolumn{3}{|c|}{ Type of $1^{\text {st }}$ colorectal cancer-related visit ${ }^{1}$} \\
\hline Late $^{2}$ event & $2545(31)$ & $506(30)$ \\
\hline (Pre)Diagnostic event & $5765(69)$ & $1166(70)$ \\
\hline \multicolumn{3}{|c|}{ Time from diagnosis to death (days) ${ }^{3}$} \\
\hline Mean [Median] & $661[453]$ & $711[466]$ \\
\hline$<=1$ month & $491(11)$ & $84(10)$ \\
\hline $1-3$ months & $472(11)$ & $90(10)$ \\
\hline$>=3$ months & $3431(78)$ & $710(80)$ \\
\hline
\end{tabular}

${ }^{1}$ Limited to healthcare visits within 1 year prior to diagnosis of colorectal cancer.

${ }^{2}$ Late event is defined as a visit that included treatment-related events (e.g., surgery, palliative care) rather than diagnostic or pre-diagnostic events (e.g.,

radiology, symptoms). Patients who did not have any colorectal cancer-related healthcare visits are also included in this group.

${ }^{3}$ Includes only patients who died on or before March 31, 2009.

was the best single administrative data source with similar completeness to the chart review alone identifying $1566(81 \%)$ of endoscopies conducted and 1300 (78\%) of the patients.

Similar to the results of the overall completeness of the single data sources, the chart review identified the most patients (40) and endoscopies (205) uniquely and the physician billing identified the most of the individual administrative data sources: 91 patients and 125 endoscopies. The combined administrative data, however, identified 133 patients (9\%) and 431 endoscopies (22\%) that were not found in the chart review.

Table 2 Summary of endoscopy information from the chart review

\begin{tabular}{lr}
\hline Description & N (\%) \\
\hline \# of Endoscopies & $1506(100)$ \\
\hline Type of Endoscopy & $1068(71)$ \\
\hline Colonoscopy & $438(29)$ \\
\hline Sigmoidoscopy & $1406(93)$ \\
\hline Results & $55(4)$ \\
\hline Cancer & $45(3)$ \\
\hline Not Cancer & \\
\hline Suspicious & $979(65)$ \\
\hline Source of Information & $527(35)$ \\
\hline Pathology Report &
\end{tabular}

Patients identified in the hospital inpatient data tended to be older and have higher stage than those identified in the other data sources: $25 \%$ of patients with an endoscopy in the inpatient data were 80 years of age or older compared to $15-20 \%$ in the other single data sources and $33 \%$ had stage IV disease compared to $16-19 \%$ in the other single data sources.

Of the 1732 endoscopies identified in the combined administrative dataset, 1289 (74\%) were found in the physician billing plus at least one of the hospital datasets and 1254 (97\%) of these had an exact match for the date of the procedure (not shown in the tables), illustrating nearperfect agreement between the physician billing and hospital data with respect to dates of endoscopy procedures.

Table 4 describes the level of agreement between data sources with respect to number of patients who had an endoscopy procedure and number of endoscopies identified. The highest level of agreement was between the chart review and combined administrative data with $90 \%$ agreement on patients identified (or not) with endoscopy and $71 \%$ agreement on endoscopies identified (or not). Agreement between physician billing and chart review was only slightly less at $85 \%$ for the patient level and $69 \%$ at the endoscopy level. The lowest agreement was between the hospital inpatient and outpatient data which was $26 \%$ at the patient level and 34\% at the endoscopy level. Most of the agreement at both the patient and endoscopy levels between these two data sources was due to the "no" cells, that is, 384 of the 443 patients (87\%) 
Table 3 Total number of patients and endoscopies identified by different data sources

\begin{tabular}{|c|c|c|c|c|}
\hline Data Source & $\frac{\text { All Patients }}{\mathrm{n}(\%)}$ & $\frac{\text { Patients with Endoscopy }}{n(\%)^{1}}$ & $\frac{\text { Patients without Endoscopy }}{n(\%)^{1}}$ & $\frac{\text { Total Number of Endoscopies }}{\mathrm{n}(\%)^{2}}$ \\
\hline & $1672(100)$ & & & \\
\hline Gold Standard & & $1443(86)$ & $229(14)$ & $1937(100)$ \\
\hline Chart Review & & $1310(78)$ & $362(22)$ & $1506(78)$ \\
\hline Unique $^{3}$ & & $40(3)$ & & $205(11)$ \\
\hline Physician Billing & & $1300(78)$ & $372(22)$ & $1566(81)$ \\
\hline Unique $^{3}$ & & $24(2)$ & & $152(8)$ \\
\hline Unique admin data ${ }^{4}$ & & $91(7)$ & & $125(6)$ \\
\hline Hospital Inpatient & & $326(19)$ & $1346(81)$ & $354(18)$ \\
\hline Unique $^{3}$ & & $9(<1)$ & & $33(2)$ \\
\hline Unique admin data ${ }^{4}$ & & $56(4)$ & & $39(2)$ \\
\hline Hospital Outpatient & & $1021(61)$ & $651(39)$ & $1126(58)$ \\
\hline Unique $^{3}$ & & $4(<1)$ & & $30(2)$ \\
\hline Unique admin data ${ }^{4}$ & & $32(2)$ & & $63(3)$ \\
\hline Combined Administrative & & $1403(84)$ & $269(16)$ & $1732(89)$ \\
\hline Unique $^{3}$ & & $133(9)$ & & $431(22)$ \\
\hline
\end{tabular}

${ }^{1}$ Percent based on all patients included in the study ( $\left.n=1443\right)$.

${ }^{2}$ Percent based on total number of endoscopies identified in gold standard $(n=1937)$.

${ }^{3}$ Number (percentage) identified in one and only one data source.

${ }^{4}$ Number (percentage) identified in one and only one of the administrative data sources, may also be identified from the chart review.

for which there was agreement did not have an endoscopy in either data source. Agreement between the physician billing and hospital inpatient was only slightly better at $37 \%$ for both patient and endoscopy level, however, the agreement was roughly equally split due to consistency in identifying patients who had (283 patients) or did not have (329 patients) an endoscopy.

In order to assess the likelihood that endoscopies were missed, even in the Gold Standard, clinical characteristics and health care service utilization were compared between patients who had an endoscopy $(n=1442)$ to those who did not $(\mathrm{n}=230)$ according to the Gold Standard. Results are shown in Table 5. Patients who did not have a record of endoscopy were more likely to be diagnosed with stage IV disease $(\mathrm{P}<0.0001)$, had shorter survival from diagnosis $(\mathrm{P}<0.0001)$, and were more likely for their first colorectal-related health care visit in the year prior to their diagnosis to be a "late" event $(P<0.0001)$ than those who had an endoscopy record. "Late" events were defined as visits that involved only services expected after cancer diagnosis has been made, such as surgery or palliative care, and did not include any expected pre-diagnostic services such as endoscopy, radiology, or presentation with symptoms.

\section{Discussion}

The purpose of this study was to determine the completeness and accuracy (with respect to dates) of various administrative data sources in identifying endoscopies in the year prior to colorectal cancer diagnosis. The findings of

Table 4 Number and percent agreement of patients and endoscopies across data sources

\begin{tabular}{|c|c|c|c|c|}
\hline & Physician Billing & Hospital Inpatient & Hospital Outpatient & Combined Administrative \\
\hline & n (\%) & n (\%) & n (\%) & n (\%) \\
\hline \multicolumn{5}{|c|}{ Chart Review } \\
\hline Patient & $1422(85)$ & $618(37)$ & $1225(73)$ & $1499(90)$ \\
\hline Endoscopy & $1790(69)$ & $860(40)$ & $1382(64)$ & $1530(71)$ \\
\hline \multicolumn{5}{|c|}{ Physician Billing } \\
\hline Patient & & $612(37)$ & $1269(76)$ & \\
\hline Endoscopy & & $808(37)$ & $1538(71)$ & \\
\hline \multicolumn{5}{|c|}{ Hospital Inpatient } \\
\hline Patient & & & $443(26)$ & \\
\hline Endoscopy & & & $736(34)$ & \\
\hline
\end{tabular}


Table 5 Patient characteristics of those who had an endoscopy prior to colorectal cancer diagnosis compared to those who did not in the Gold Standard dataset

\begin{tabular}{|c|c|c|c|}
\hline Characteristics & $\begin{array}{l}\text { Did not have } \\
\text { endoscopy } \mathrm{n}(\%)\end{array}$ & $\begin{array}{l}\text { Had endoscopy } \\
\text { n (\%) }\end{array}$ & $P$ value \\
\hline Total Number & $229(100)$ & $1443(100)$ & \\
\hline Age at diagnosis & & & 0.24 \\
\hline Mean [Median] & 70 [72] & 68 [69] & \\
\hline$<=65$ & $84(37)$ & $559(39)$ & \\
\hline $66-74$ & $53(23)$ & $384(26)$ & \\
\hline$>=75$ & $92(40)$ & $500(35)$ & \\
\hline Stage at diagnosis & & & $<.0001$ \\
\hline$|-|||$ & $110(48)$ & $1035(72)$ & \\
\hline IV or Unknown & $119(52)$ & $408(28)$ & \\
\hline Type of $1^{\text {st }}$ colorectal cancer-related visit ${ }^{1}$ & & & $<.0001$ \\
\hline Late $^{2}$ event & $104(45)$ & $402(28)$ & \\
\hline (Pre)Diagnostic event & $125(55)$ & $1041(72)$ & \\
\hline Time from diagnosis to death (days) $^{3}$ & & & $<.0001$ \\
\hline Mean [Median] & $467[200]$ & $771[546]$ & \\
\hline$<=1$ month & $41(24)$ & $43(6)$ & \\
\hline 1 - 3 months & $20(11)$ & $70(10)$ & \\
\hline$>=3$ months & $111(64)$ & 599 (84) & \\
\hline
\end{tabular}

the study support the use of physician billing alone or combined with hospital inpatient and outpatient data as reasonable data sources for identifying patients who have had at least one endoscopy in the year prior to colorectal cancer diagnosis but a combination of hospital and physician billing data is recommended to identify the total number of endoscopies received. This conclusion is restricted to the setting in which the majority of physicians performing endoscopy are remunerated on a fee-forservice basis in the single-payer health care system and/or in which salaried physicians submit claims for procedures performed. Hospital data alone are not good sources for this information because a significant number and percentage of endoscopies occur outside the hospital.

Physician billing data are created for the purpose of remunerating physicians who are paid on a fee-for-service schedule. The completeness of the data is likely to be high if specific fee code exists for a well-defined procedure (such as endoscopy) and physicians have the incentive to record the procedure accurately in their claim for their fee reimbursement. Accuracy of the physician billing data, therefore, is subject to the fee code policy. The results of studies based on physician billing data could easily be misinterpreted if certain procedure codes are unknowingly under or over claimed due to variances in reimbursement for related and/or similar procedures. Caution is, therefore, needed in the conduct and interpretation of studies based on physician billing data; strong understanding of the way in which physicians use billing codes and the percentage of physicians who perform the procedure of interest that bill for it is needed. Validation of the data is also critical.

One of the shortcomings to our method of validation was the lack of independence between our gold standard dataset and our comparison data sets. Our study did not evaluate the accuracy of the endoscopy with respect to type of exam (colonoscopy vs. sigmoidoscopy) or reason for exam (screening vs. diagnosis), however, a few studies have done so. Not surprisingly, they have all found that administrative data are not adequate for assessing this level of specificity with respect to type or reason for exam [9,27-29]. For instance, Schenck et al. found Medicare claims to be accurate for identifying endoscopies but not for distinguishing screening from diagnostic tests. This is at least in part due to the absence of billing codes that are specific to screening tests but even if implemented, the fee code would need to be comparable to the diagnostic fee code in order to provide physicians incentive to use it.

As mentioned, it is expected that patients with colorectal cancer would have at least one endoscopy procedure prior to their diagnosis as endoscopy is the most common definitive diagnostic procedure. Fourteen percent of the patients in the study, however, did not have 
any endoscopy record in the gold standard. To examine the likelihood that endoscopies were missed, even in the gold standard, we explored clinical characteristics and other health service utilization of patients who did not have an endoscopy procedure identified in any of the data sources $(n=230)$. About half of these patients presented with stage IV disease and about half had at least one colorectal-related symptom at a healthcare visit within one year prior to their colorectal cancer diagnosis. One would expect that patients who had colorectalrelated symptoms prior to diagnosis would have had an endoscopy so it is possible that endoscopies for these patients $(n=110)$ were incorrectly missed. Alternatively, it is possible that some of these patients were diagnosed via an alternative route such as by a CT scan that identified metastatic disease or as an emergency patient that went straight to surgery. The high percentage of patients with stage IV disease who did not have an endoscopy recorded makes these alternative diagnostic routes likely. Additionally, some patients may have had an endoscopy in another province. A minority of cancer patients receives treatment outside the province and some may receive some or all of their diagnostic work-up outside the province as well. Given these possible scenarios, it is likely that at least 100 to 125 patients $(5-7.5 \%)$ of the total patient cohort did not have an endoscopy at all or in Alberta prior to their colorectal cancer diagnosis. The patients who received endoscopies within Alberta as part of the process in diagnosing colorectal cancer, therefore, seem to have been properly identified in the gold standard created for the study combining chart review and administrative data sources.

\section{Conclusion}

Usually the gold standard for validation of administrative data is a disease registry database or medical records $[10,12,18,22,30,31]$. We chose to combine the information from chart review and each administrative dataset because of recognized limitations to each data source on its own for identifying endoscopies and potential inaccuracies of dates. Additionally, because it is expected that all but a small minority of patients diagnosed with colorectal cancer would have at least one endoscopy in the year prior to diagnosis we were confident that the probability of a false positive in any data source would be negligible. The findings of this study with respect to completeness and accuracy of data sources should be generalizable across Canada and in other jurisdictions in which endoscopies are reimbursed via feefor-service and similar datasets exist. This is because in Canada, the inpatient and outpatient databases are standardized nationally, even though they are prepared provincially, and have ongoing quality assessments made to them nationally [32]. Furthermore, we expect the methodology for creating a gold standard to be appropriate in similar scenarios in which the procedure is well-defined, is expected to occur in the majority of the population, and for which a true gold standard does not exist. In the absence of an official registry database for endoscopy procedures, physician billing combined with hospital data is the most complete source of information to identify endoscopies.

\section{Additional file}

\section{Additional file 1: Colorectal endoscopy procedure codes.}

\section{Abbreviations}

AHCIP: Alberta Health Care Insurance Program; ARP: Alternate Relationship Plan; ICD-O: International Classification of Diseases for Oncology; ULI: Unique lifetime identifier.

\section{Competing interests}

The authors declare that they have no competing interests.

\section{Authors' contributions}

$\mathrm{XL}$ and SH contributed to the statistical analysis, interpretation of data and drafting of the manuscript. $\mathrm{RH}$ helped obtain funding, contributed to the study design; interpretation of data; critical revision of the manuscript for important intellectual content; and provided clinical input. JF contributed to the statistical analysis. MW was the overall supervisor of the study and as such contributed in all aspects of the study including obtaining funding, overseeing the analysis, interpretation, critical revision and final approval of the manuscript. All authors read and approved the final manuscript.

\section{Acknowledgements}

The authors thank Angela Bella for assistance in formatting the final manuscript. This study was funded in part by the Canadian Cancer Society and the Alberta Cancer Foundation. Dr. Robert Hilsden is an Alberta Innovates - Health Solutions Health Scholar.

\section{Author details}

${ }^{1}$ Division of Community Oncology, Cancer Care, Alberta Health Services, Edmonton, Alberta, Canada. ${ }^{2}$ Department of Community Health Sciences and Department of Medicine, University of Calgary, Calgary, Alberta, Canada. ${ }^{3}$ Department of Public Health Sciences, School of Public Health, University of Alberta, 1500 Sun Life Place, 1012399 Street NW, Edmonton, Alberta T5J $3 \mathrm{H} 1$, Canada. ${ }^{4}$ Present address: Department of Mathematics and Statistics, University of Winnipeg, Winnipeg, Canada.

Received: 29 February 2012 Accepted: 5 October 2012 Published: 13 October 2012

\section{References}

1. lezzoni LI: Assessing quality using administrative data. Ann Intern Med 1997, 127:666-74.

2. Logan JR, Lieberman DA: The use of databases and registries to enhance colonoscopy quality. Gastrointest Endosc Clin N Am 2010, 20:717-34.

3. Peabody JW, Luck J, Jain S, Bertenthal D, Glassman P: Assessing the accuracy of administrative data in health information systems. Medical Care 2004, 42:1066-72.

4. Pinfold SP, Goel V, Sawka C: Quality of hospital discharge and physician data for type of breast cancer surgery. Medical Care 2000, 38:99-107.

5. Smalley W: Administrative data and measurement of colonoscopy quality: not ready for prime time? Gastrointest Endosc 2011, 73:454-5.

6. Tollefson MK, Gettman MT, Karnes RJ, Frank I: Administrative data sets are inaccurate for assessing functional outcomes after radical prostatectomy. J Urol 2011, 185:1686-90.

7. Turner D, Hildebrand KJ, Fradette KSL: Same question, different data source, different answers? Data source agreement for surgical procedures on women with breast cancer. Healthc Policy 2007, 3:46-54.

8. Woodworth GF, Baird CJ, Garces-Ambrossi G, Tonascia J, Tamargo RJ: Inaccuracy of the administrative database: comparative analysis of two 
databases for the diagnosis and treatment of intracranial aneurysms. Neurosurgery 2009, 65:251-6.

9. Wyse JM, Joseph L, Barkun AN, Sewitch MJ: Accuracy of administrative claims data for polypectomy. CMAJ 2011, 183:E743-E747.

10. Abraham NS, Gossey JT, Davila JA, Al-Oudat S, Kramer JK: Receipt of recommended therapy by patients with advanced colorectal cancer. Am J Gastroenterol 2006, 101:1320-8.

11. Amed S, Vanderloo SE, Metzger D, Collet JP, Reimer K, McCrea P, Johnson $J A$ : Validation of diabetes case definitions using administrative claims data. Diabet Med 2011, 28:424-7.

12. Andrade SE, Gurwitz JH, Chan KA, Donahue JG, Beck A, Boles M, Buist DS, Goodman M, LaCroix AZ, Levin TR, Platt R: Validation of diagnoses of peptic ulcers and bleeding from administrative databases: a multi-health maintenance organization study. J Clin Epidemio/ 2002, 55:310-3.

13. Dodds L, Spencer A, Shea S, Fell D, Armson BA, Allen AC, Bryson S: Validity of autism diagnoses using administrative health data. Chronic Dis Can 2009, 29:102-7.

14. Lix L, Yogendran M, Burchill C, Metge C, McKeen N, Moore D, Bond R: Defining and validating chronic diseases: an administrative data approach. Winnipeg: Manitoba Centre for Health Policy; 2006. Ref Type: Generic.

15. Lo-Ciganic W, Zgibor JC, Ruppert K, Arena VC, Stone RA: Identifying type 1 and type 2 diabetic cases using administrative data: a tree-structured model. J Diabetes Sci Technol 2011, 5:486-93.

16. Lopushinsky SR, Covarrubia KA, Rabeneck L, Austin PC, Urbach DR: Accuracy of administrative health data for the diagnosis of upper gastrointestinal diseases. Surg Endosc 2007, 21:1733-7.

17. Nattinger AB, Laud PW, Bajorunaite R, Sparapani RA, Freeman JL: An algorithm for the use of Medicare claims data to identify women with incident breast cancer. Health Serv Res 2004, 39:1733-49.

18. Ramsey S, Mandelson M, Etzioni R, Harrison R, Smith R, Taplin S: Can administrative data identify incident cases of colorectal cancer? a comparison of two health plans. Health Serv Outcomes Res Methodol 2004, 5:27-37.

19. Tu K, Campbell NR, Chen ZL, Cauch-Dudek KJ, McAlister FA: Accuracy of administrative databases in identifying patients with hypertension. Open Med 2007, 1:e18-e26.

20. Zgibor JC, Orchard TJ, Saul M, Piatt G, Ruppert K, Stewart A, Siminerio LM: Developing and validating a diabetes database in a large health system. Diabetes Res Clin Pract 2007, 75:313-9.

21. Cooper GS, Schultz L, Simpkins J, Lafata JE: The utility of administrative data for measuring adherence to cancer surveillance care guidelines. Med Care 2007, 45:66-72

22. Quan H, Parsons GA, Ghali WA: Assessing accuracy of diagnosis-type indicators for flagging complications in administrative data. J Clin Epidemiol 2004, 57:366-72.

23. Hilsden RJ, Bryant HE, Sutherland LR, Brasher PM, Fields AL: A retrospective study on the use of post-operative colonoscopy following potentially curative surgery for colorectal cancer in a Canadian province. BMC Cancer 2004, 4:14.

24. Peters D, Chen C, Markson LE, Len-Ramey FC, Vollmer WM: Using an asthma control questionnaire and administrative data to predict health-care utilization. Chest 2006, 129:918-924.

25. Fritz A, Percy C, Jack A, Shanmugaratnam K, Sobin L, Parkin DM, et al: (eds): International Classification of Diseases for Oncology. 3rd edition. Geneva, Switzerland: World Health Organization; 2000.

26. Collaborative Staging Task Force of the American Joint Committee on Cancer: Collaborative Staging Manual and Coding Instructions, version 01.04.00: Jointly published by American Joint Committee on Cancer (Chicago, IL) and U.S. Department of Health and Human Services (Bethesda, MD); 2004. NIH Publication Number 04-5496. Incorporates updates through September 8, 2006.

27. Fisher DA, Grubber JM, Castor JM, Coffman CJ: Ascertainment of colonoscopy indication using administrative data. Dig Dis Sci 2010, 55:1721-5.

28. Haque R, Chiu V, Mehta KR, Geiger AM: An automated data algorithm to distinguish screening and diagnostic colorectal cancer endoscopy exams. J Natl Cancer Inst Monogr 2005, 35:116-118.

29. Schenck AP, Klabunde CN, Warren JL, Peacock S, Davis WW. Hawley ST, Pignone M, Ransohoff DF: Data sources for measuring colorectal endoscopy use among Medicare enrollees. Cancer Epidemiol Biomarkers Prev 2007, 16:2118-27.
30. Goff SL, Feld A, Andrade SE, Mahoney L, Beaton SJ, Boudreau DM, Davis RL, Goodman M, Hartsfield CL, Platt R, Roblin D, Smith D, Yood MU, Dodd K, Gurwitz JH: Administrative data used to identify patients with irritable bowel syndrome. J Clin Epidemiol 2008, 61:617-21.

31. Miller DC, Saigal CS, Warren JL, Leventhal M, Deapen D, Banerjee M, Lai J, Hanley J, Litwin MS: External validation of a claims-based algorithm for classifying kidney-cancer surgeries. BMC Health Serv Res 2009, 9:92.

32. Canadian Institute for Health Information. http:/www.cihi.ca/CIHI-ext-portal/ internet/EN/TabbedContent/standards+and+data+submission/data+quality/ cihi021513.

doi:10.1186/1472-6963-12-358

Cite this article as: Li et al.: Validation of administrative data sources for endoscopy utilization in colorectal cancer diagnosis. BMC Health Services Research 2012 12:358.

\section{Submit your next manuscript to BioMed Central and take full advantage of:}

- Convenient online submission

- Thorough peer review

- No space constraints or color figure charges

- Immediate publication on acceptance

- Inclusion in PubMed, CAS, Scopus and Google Scholar

- Research which is freely available for redistribution

Submit your manuscript at www.biomedcentral.com/submit
C Biomed Central 